\title{
Aberrant Expression of N-Methylpurine-DNA Glycosylase Influences Patient Survival in Malignant Gliomas
}

\author{
Ce Liu, ${ }^{1}$ Yanyang Tu, ${ }^{2}$ Jun Yuan, ${ }^{3}$ Xinggang Mao, ${ }^{4}$ Shiming He, ${ }^{5}$ Liang Wang, \\ Guoqiang Fu, ${ }^{2}$ Jianhai Zong, ${ }^{5}$ and Yongsheng Zhang ${ }^{3}$ \\ ${ }^{1}$ Department of Neurosurgery, The 309th Hospital of PLA, Beijing 100091, China \\ ${ }^{2}$ Department of Emergency, Tangdu Hospital, The Fourth Military Medical University, Xi'an 710038, China \\ ${ }^{3}$ Administrative Department, Tangdu Hospital, The Fourth Military Medical University, Xi'an 710038, China \\ ${ }^{4}$ Department of Neurosurgery, 254th Hospital of PLA, Tianjin 300142, China \\ ${ }^{5}$ Department of Neurosurgery, Tangdu Hospital, The Fourth Military Medical University, Xi'an 710038, China
}

Correspondence should be addressed to Yongsheng Zhang, zhangys_td@163.com

Received 27 November 2011; Revised 1 January 2012; Accepted 18 January 2012

Academic Editor: Paul W. Doetsch

Copyright ( 92012 Ce Liu et al. This is an open access article distributed under the Creative Commons Attribution License, which permits unrestricted use, distribution, and reproduction in any medium, provided the original work is properly cited.

\begin{abstract}
Aim. To examine the expression of N-methylpurine-DNA glycosylase (MPG) gene and protein in glioma samples with different WHO grades and its association with patients' survival. Methods. Immunohistochemistry assay, quantitative real-time PCR and Western blot analysis were carried out to investigate the expression of MPG gene and protein in 128 glioma and 10 non-neoplastic brain tissues. Results. MPG gene expression level in glioma tissues was significantly higher than that in non-neoplastic brain tissues $(P<0.001)$. Immunohistochemistry also showed that MPG protein was over-expressed in glioma tissues, which was consistent with the resutls of Western blot analysis. Additionally, the expression levels of MPG gene and protein both increase from grade I to grade IV glioma according to the results of real-time PCR, immunohistochemistry and western blot analysis. Moreover, the survival rate of MPG-positive patients was significantly lower than that of MPG-negative patients $(P<0.001)$. We further confirmed that the over-expression of MPG was a significant and independent prognostic indicator in glioma by multivariate analysis $(P<0.001)$. Conclusions. Our data showed the over-expression of MPG gene and protein in human gliomas, and also suggested for the first time that MPG be an unfavorable independent prognostic indicator for glioma patients.
\end{abstract}

\section{Introduction}

Human gliomas represent $50 \%$ to $60 \%$ of all intracranial tumors [1]. According to the World Health Organization (WHO) guidelines [2], gliomas are histologically classified into four grades: pilocytic astrocytoma (grade I), low-grade diffuse astrocytoma (grade II), anaplastic astrocytoma (grade III), and glioblastoma multiforme (GBM, grade IV). Both diagnostic technologies and therapeutic strategies have been greatly advanced, but glioma remains one of the deadliest human cancers. The 5-year survival rates of low-grade (grade I $\sim$ II) and high-grade (grade III $\sim$ IV) glioma patients in China are $75.4 \%$ and $18.2 \%$, respectively [3]. Especially, the median survival time for patients with GBM is still only 12 months [4]. Indeed, early diagnosis and prolonging survival in glioma patients remains a great challenge for clinicians in the field of neurooncology. There have been several prognostic factors for glioma patients, such as age, preoperative duration of symptoms, Karnofsky performance status (KPS) score, histologic grade, tumor necrosis, surgical resection extent, use of postoperative radiation therapy, and, probably, adjuvant chemotherapy [5]. However, these clinical parameters cannot completely account for the observed variation in survival because of the heterogeneity of glioma patients [6]. Thus, there is an urgent need to further investigate the molecular mechanisms of glioma and to identify the effective prognostic indicators for survival prediction. The DNA-base excision repair (BER) pathway is responsible for the repair of exogenous and endogenous alkylating and oxidative DNA damage, which may lead to carcinogenesis, cell death, and aging if left unrepaired [7]. The schematic diagram of BER pathway is shown as 
Figure 1. This pathway involves the recognition and removal of damaged bases by a DNA glycosylase, followed by incision of the resulting abasic (AP) site by AP endonuclease, DNA synthesis by polymerase, and strand ligation by DNA ligase [8]. Thus, the BER pathway is an important candidate for intervention into the cellular responses to DNA change. $\mathrm{N}$-methylpurine-DNA glycosylase (MPG) as a DNA repair enzyme is a main component in the BER pathway [9]. In previous study aimed at understanding the significance of initiating lesions removed by the BER pathway, Kaina et al. [10] identified the over-expression of the human MPG in Chinese hamster ovary cells. In the N-alkylpurine repair process, MPG is responsible for the glycolytic removal of the modified base, which leads to the formation of apurinic sites. Although $\mathrm{N}$-alkylpurines have not been found to be directly mutagenic, apurinic sites left by this repair process can block replication and lead to mutation [11]. MPG also participates in the repair of 8-hydroxyguanine and hypoxanthine [12]. Because of the potential role of DNA base lesions in mutagenesis and carcinogenesis, a number of studies have been performed to investigate the association of MPG with various human cancers. Cerda et al. (1998) [13] detected the increased MPG gene and protein expression in the breast cancer cells versus normal breast epithelial cells by northern analysis, southern blots, immunofluorescence, immunohistochemistry, and western blot analysis. In 2001, Sohn et al. [14] reported that the expression of MPG was increased in high-risk HPV-infected cervical neoplasias and the intracellular distribution of MPG protein was altered, suggesting a role of MPG in carcinogenesis. In an effort to improve the efficacy of cancer chemotherapy by intervening into the cellular responses to chemotherapeutic change, many researchers have been interested in the effects of MPG in tumor sensitivity to the clinical chemotherapeutic agents. As their results, MPG-overexpressing ovarian cancer [15], osteosarcoma [16], and breast cancer [17] cells are significantly more sensitive to the clinical chemotherapeutic agents, suggesting that the overexpression of MPG may be a possible gene therapy approach to sensitize tumor cells to the cell-killing effects of chemotherapeutic alkylating agents. The biological mechanism behind the increase of sensitivity to the chemotherapeutic agents in MPG overexpressing cell lines may be that the balance between glycosylase activity, leading to apurinic sites and formation of strand breaks, and subsequent excision repair processes may play an important role in determining cellular cytotoxicity and resistance to alkylating agents [13].

Of our interests, we focus on the involvement of MPG in human gliomas. Recent study has demonstrated that MPG mRNA expression was higher in astrocytic tumor tissues than in brain tissues adjacent to tumor, and MPG protein localization in immunohistochemical study was detected only in the nucleus of all tumor tissues, suggesting an MPG's role in human astrocytic tumors and raise the possibility that the altered MPG expression and intracellular localization could be associated with astrocytic tumorigenesis [18]. Tang et al. [19] further demonstrated that MPG overexpression, together with the inhibition of BER, could sensitize glioma cells to the alkylating agent. However, little is known about the expression level of MPG in human gliomas with different clinical grades and its prognostic significance. To address this problem, we used quantitative real-time PCR, immunohistochemistry assay, and western blot analysis to investigate the expression pattern of MPG gene and protein in glioma specimens and normal control brain tissues. Next, we analyzed the relationship between MPG expression and the glioma stage as well as the survival of patients.

\section{Materials and Methods}

2.1. Patients and Tissue Samples. This study was approved by the Research Ethics Committee of the Institute for functional neurosurgery P.L.A, TangDu Hospital, Fourth Military Medical University, Xi'an, P.R. China. Written informed consent was obtained from all of the patients. All specimens were handled and made anonymous according to the ethical and legal standards.

A total of 128 formalin-fixed, paraffin-embedded specimens of gliomas resected between 2000 and 2010 were retrieved from the archives of the Pathology Department of Tangdu Hospital, Fourth Military Medical University, P.R. China. All the slides were reevaluated according to WHO classifications [2] by two pathologists, with differences resolved by careful discussion. A total of 76 males and 52 females $(1.46: 1)$ were enrolled in this study, and the median age was 42 years (range, 12-71). Thirty-two of the 128 gliomas were classified as low-grade [18 pilocytic astrocytomas (WHO I) and 14 diffuse astrocytomas (WHO II)], and 96 were classified as high-grade gliomas [38 anaplasia astrocytomas (WHO III), and 58 primary glioblastomas (WHO IV)]. None of the patients had received chemotherapy or radiotherapy prior to surgery. The clinicopathological features, and the treatment strategies of all the patients were indicated in Table 1. Paraffin and snap-frozen sections of nonneoplastic brain tissues from 10 patients with intractable epilepsy were also included as controls. Five-year followup was performed, and all patients had complete followup until death. Overall survival time was calculated from the date of the initial surgical operation to death. Patients, who died of diseases not directly related to their gliomas or due to unexpected events, were excluded from this study. In addition, 20 glioma specimens [ 5 pilocytic astrocytomas (WHO I), 3 diffuse astrocytomas (WHO II), 3 anaplasia astrocytomas (WHO III), and 9 primary glioblastomas (WHO IV)] were snap-frozen in liquid nitrogen and stored at $-80^{\circ} \mathrm{C}$ following surgery for mRNA and protein isolation.

2.2. Quantitative Real-Time PCR. Total RNA purified from all 20 glioma tissues and 10 control brain tissues was prepared and reverse transcribed. Real-time monitoring of polymerase chain reactions (PCRs) was performed using the ABI 7900HT (Idaho Technology, Idaho Falls, ID, USA). cDNA was synthesized using random primers and Oligo $18 \mathrm{dT}$ and Superscript II Reverse Transcriptase (Invitrogen). Gene expression was determined using the SYBR green I dye (Biogene), which binds preferentially to double-stranded DNA, and $10 \mathrm{ng}$ of template. Fluorescence signals, which are 


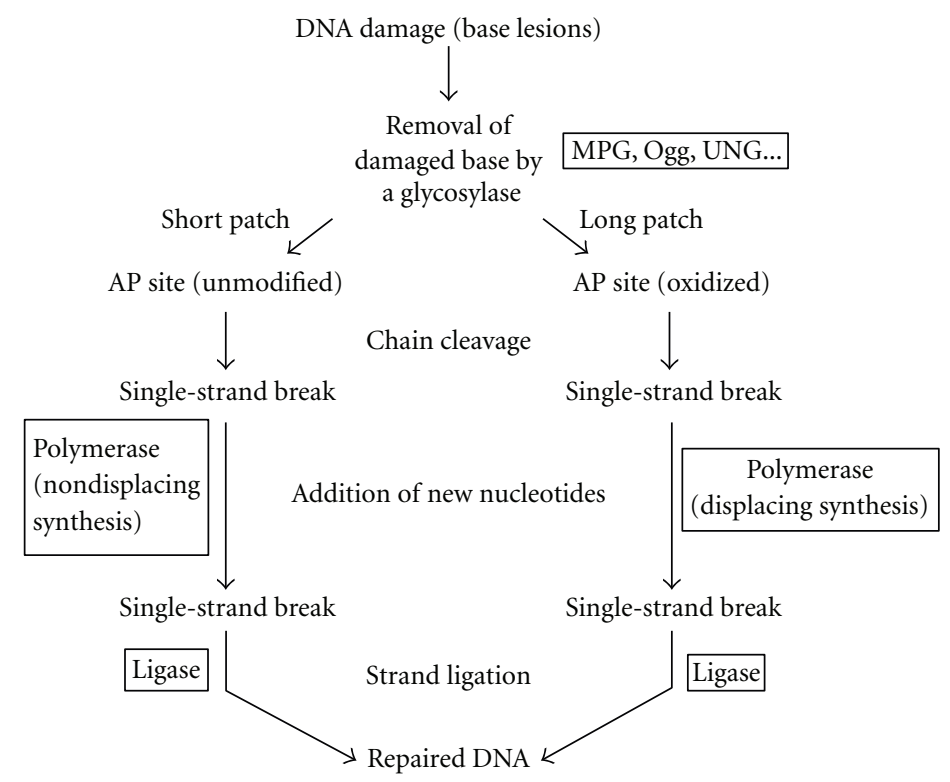

FIGURE 1: The schematic diagram of base excision repair (BER) pathway for DNA repair throughout the cell cycle. BER is important for removing damaged bases. BER is initiated by DNA glycosylases (e.g., MPG), which recognize and remove specific damaged or inappropriate bases, forming AP sites. These are then cleaved by an AP endonuclease. The resulting single-strand break can then be processed by either short-patch or long-patch BER.

proportional to the concentration of the PCR product, are measured at the end of each cycle and immediately displayed on a computer screen, permitting real-time monitoring of the PCR. The reaction is characterized by the point during cycling when amplification of PCR products is first detected, rather than the amount of PCR product accumulated after a fixed number of cycles. The higher the starting quantity of the template, the earlier a significant increase in fluorescence is observed. The threshold cycle is defined as the fractional cycle number at which fluorescence passes a fixed threshold above the baseline. The primers 5'-GTC CTA GTC CGG CGA CTT CC- $3^{\prime}$ and $5^{\prime}$-CTT GTC TGG GCA GGC CCT TTG C-3' were used to amplify 603-bp transcripts of MPG, and the primers $5^{\prime}$-GGT GGC TTT TAG GAT GGC AAG-3' and 5'-ACT GGA ACG GTG AAG GTG ACA G-3' were used to amplify 161-bp transcripts of $\beta$-actin. All primers were synthesized by Sangon Co. (Shanghai, China). The PCR profile consisted of an initial melting step of $1 \mathrm{~min}$ at $94^{\circ} \mathrm{C}$, followed by 38 cycles of $15 \mathrm{~s}$ at $94^{\circ} \mathrm{C}, 15 \mathrm{~s}$ at $56^{\circ} \mathrm{C}$, and $45 \mathrm{~s}$ at $72^{\circ} \mathrm{C}$, and a final elongation step of $10 \mathrm{~min}$ at $72^{\circ} \mathrm{C}$.

Fluorescence data were converted into cycle threshold measurements using the SDS system software and exported to Microsoft Excel. MPG mRNA levels were compared to $\beta$ actin. Thermal dissociation plots were examined for biphasic melting curves, indicative of whether primer dimers or other nonspecific products could be contributing to the amplification signal.

2.3. Western Blot Analysis. Twenty glioma tissues and 10 control brain tissues were homogenized in lysis buffer [PBS, 1\% nonidet P-40 (NP-40), 0.5\% sodium deoxycholate, $0.1 \%$ sodium dodecyl sulfate (SDS), $100 \mathrm{ug} / \mathrm{mL}$ aprotinin,
$100 \mu \mathrm{g} / \mathrm{mL}$ phenylmethylsulfonyl fluoride (PMSF), Sodium orthovanadate] at $4^{\circ} \mathrm{C}$ throughout all procedures, and sonicated for $70 \mathrm{~s}$, then add $300 \mu \mathrm{g}$ PMSF per gram of tissue and incubate on ice for $30 \mathrm{~min}$, followed by centrifugation at $15,000 \mathrm{rpm}$ for $20 \mathrm{~min}$ at $4^{\circ} \mathrm{C}$. The protein content was determined according to Bradford's method [20], with bovine serum albumin used as a standard. Protein samples $(30 \mu \mathrm{g})$ were boiled with $2 \times$ sample buffer containing $5 \% \beta$-mercaptoethanol for $5 \mathrm{~min}$, separated by size on $15 \%$ polyacrylamide gel under SDS denaturing conditions, and transferred to a nitrocellulose membrane at $90 \mathrm{~V}$ for $2 \mathrm{~h}$. The nitrocellulose membranes were stained with ponceau $S$ to assess the efficiency of transfer. Nonspecific binding was blocked by incubation in block buffer ( $5 \%$ nonfat dry milk, $0.05 \%$ Tween-20, $1 \times$ tris-Cl-buffered saline) overnight at $4^{\circ} \mathrm{C}$, The membranes were hybridized with a mouse monoclonal antibody to MPG (ab55461; Abcam), then incubated with a horseradish peroxidase-labeled goat anti-mouse IgG (1:500). The bound secondary antibody was detected by enhanced chemiluminescence (Amersham Life Science, Little Chalfont, UK). Housekeeping protein $\beta$ actin was used as a loading control. Positive immunoreactive bands were quantified densitometrically (Leica Q500IW image analysis system) and expressed as ratio of MPG to $\beta$ actin in optical density units.

2.4. Immunohistochemistry Assay. Immunohistochemical assay was performed using the conventional immunoperoxidase technique according to the protocol of the Department of Neurosurgery, Institute for functional neurosurgery P.L.A, TangDu Hospital, Fourth Military Medical University, Xi'an, P.R. China. Briefly, following peroxidase blocking with $0.3 \%$ 
TABLE 1: MPG expression in human glioma and nonneoplastic brain tissues with different clinical-pathological features.

\begin{tabular}{|c|c|c|c|c|c|c|}
\hline \multirow{2}{*}{ Clinicopathological features } & \multirow{2}{*}{ No. of cases } & \multicolumn{4}{|c|}{$\operatorname{MPG}(n, \%)$} & \multirow{2}{*}{$P$} \\
\hline & & - & + & ++ & +++ & \\
\hline \multicolumn{7}{|l|}{ Human glioma tissues } \\
\hline \multicolumn{7}{|l|}{ WHO grade } \\
\hline I & 18 & $14(77.8)$ & $4(22.2)$ & 0 & 0 & \multirow{4}{*}{0.002} \\
\hline II & 14 & $8(57.1)$ & $4(28.6)$ & $2(14.3)$ & 0 & \\
\hline III & 38 & $4(10.5)$ & $19(50.0)$ & $13(34.2)$ & $2(5.3)$ & \\
\hline IV & 58 & 0 & 0 & $15(25.9)$ & $43(74.1)$ & \\
\hline \multicolumn{7}{|l|}{ Age } \\
\hline$<55$ & 52 & $7(13.5)$ & $11(21.2)$ & $14(26.9)$ & $20(38.5)$ & \multirow{2}{*}{ NS } \\
\hline$\geq 55$ & 76 & $19(25.0)$ & $16(21.1)$ & $16(21.1)$ & $25(32.9)$ & \\
\hline \multicolumn{7}{|l|}{ Gender } \\
\hline Male & 76 & $19(25.0)$ & $19(25.0)$ & $15(19.7)$ & $23(30.2)$ & \multirow{2}{*}{ NS } \\
\hline Female & 52 & $7(13.5)$ & $8(15.4)$ & $15(28.8)$ & $22(42.3)$ & \\
\hline \multicolumn{7}{|l|}{ KPS } \\
\hline$<80$ & 78 & $6(7.7)$ & $15(19.2)$ & $22(28.2)$ & $35(44.9)$ & \multirow{2}{*}{0.01} \\
\hline$\geq 80$ & 50 & $20(40.0)$ & $12(24.0)$ & $8(16.0)$ & $10(20.0)$ & \\
\hline \multicolumn{7}{|l|}{ Surgery } \\
\hline Gross total resection & 98 & $18(18.4)$ & $19(19.4)$ & $28(28.6)$ & $33(33.7)$ & \multirow{3}{*}{ NS } \\
\hline Partial resection & 24 & $6(25.0)$ & $6(25.0)$ & $1(4.2)$ & $11(45.8)$ & \\
\hline Biopsy & 6 & $2(33.3)$ & $2(33.3)$ & $1(16.7)$ & $1(16.7)$ & \\
\hline \multicolumn{7}{|l|}{ Nonneoplastic brain tissues } \\
\hline \multicolumn{7}{|l|}{ Age } \\
\hline$<55$ & 4 & $3(75.0)$ & $1(25.0)$ & 0 & 0 & \multirow{2}{*}{ NS } \\
\hline$\geq 55$ & 6 & $5(83.3)$ & $1(16.7)$ & 0 & 0 & \\
\hline \multicolumn{7}{|l|}{ Gender } \\
\hline Male & 6 & $5(83.3)$ & $1(16.7)$ & 0 & 0 & \multirow{2}{*}{ NS } \\
\hline Female & 4 & $3(75.0)$ & $1(25.0)$ & 0 & 0 & \\
\hline
\end{tabular}

$\mathrm{H}_{2} \mathrm{O}_{2}$ /methanol for 30 min, specimens were blocked with phosphate-buffered saline (PBS) containing 5\% normal horse serum (Vector Laboratories Inc., Burlingame, CA, USA). All incubations with a mouse monoclonal antibody to MPG (ab55461; Abcam) at 1:500 dilution were carried out overnight at $4^{\circ} \mathrm{C}$. Then the specimens were briefly washed in PBS and incubated at room temperature with the anti-mouse antibody and avidin-biotin peroxidase (Vector Laboratories Inc., Burlingame, CA, USA). The specimens were then washed in PBS and color developed by diaminobenzidine solution (Dako Corporation, Carpinteria, CA, USA). After washing with water, specimens were counterstained with Meyer's hematoxylin (Sigma Chemical Co., St Louis, MO, USA). Nonneoplastic brain tissues were used as control tissues, and nonimmune IgG was also used as negative control antibody for immunohistochemical staining.

Stained sections were observed under a microscope. Immunostaining was scored by two independent experienced pathologists, who were blinded to the clinicopathologic parameters and clinical outcomes of the patients. An immunoreactivity score system was applied as described previously [17]. The extensional standard was (1) the number of positively stained cells $<5 \%$ scored $0,6-25 \%$ scored 1, 26-50\% scored 2, 51-75\% scored 3, >75\% scored 4 ; (2) intensity of stain: colorless scored 0 , pallide-flavens scored 1, yellow scored 2, brown scored 3. Multiply (1) and (2). The staining score was stratified as - (0 score, absent), $+(1-4$ score, weak $),++(5-8$ score, moderate $)$, and $+++(9-$ 12 score, strong) according to the proportion and intensity of positively stained cancer cells. Specimens were rescored if difference of scores from two pathologists was $>3$.

2.5. Statistical Analysis. All computations were carried out using the software of SPSS version13.0 for Windows (SPSS Inc, IL, USA). The rank sum test was used to analyze the ranked data. The measurement data were analyzed by oneway ANOVA. Randomized block design ANOVA was used to analyze the statistical difference among different tissue types. In the analysis of glioma morbidity for all patients, we used the Kaplan-Meier estimator and univariate Cox regression analysis to assess the marginal effect of each factor. The differences between groups were tested by log-rank analyses. The joint effect of different factors was assessed using multivariate Cox regression. A Spearman's analysis was carried out to analyze the correlation between MPG mRNA 
and protein expression levels. Differences were considered statistically significant when $P$ was less than 0.05 .

\section{Results}

3.1. Quantitative Analysis of MPG Gene Expression Based on WHO Grades in Gliomas. The gene expression of MPG normalized to $\beta$-actin in 20 glioma and 10 control brain tissues was detected by real-time PCR. As shown in Figure 2, the expression levels of MPG gene in human glioma tissues were conspicuously higher than those in control brain tissues $(P<0.001)$. Additionally, we also analyzed the expression of MPG gene based on WHO grade. Interestingly, MPG gene expression increased with the advancement of WHO grades from I to IV $(P<0.001)$.

\subsection{Quantitative Analysis of MPG Protein Expression Based} on WHO Grades in Gliomas. The protein expression of MPG normalized to $\beta$-actin in 20 glioma and 10 control brain tissues was also detected by Western blot analysis. As the results, the expression levels of MPG protein tended to increase from the glioma to the normal tissue (Figures 3(a) and $3(\mathrm{c}))$. We also investigated whether the expression of MPG correlated with the WHO grade. MPG expression was highest in grade IV and lowest in grade I (Figures 3(b) and 3(c)), indicating a close correlation of MPG protein expression with WHO grade. There was a significant positive correlation between the expression of MPG gene and protein expression levels from the same glioma tissues ( $\mathrm{rs}=0.82$, $P<0.001)$.

\subsection{MPG Protein Expression in Glioma Tissues by Immuno-} histochemistry Assay and Survival Analysis. The expression and the subcellular localization of MPG protein in 10 nonneoplastic brain and 128 glioma tissues were detected by immunohistochemistry assay. As shown in Figure 4, the positive staining for MPG was mainly observed in the nuclei of tumor cells in glioma tissues, which was consistent with the previous study of wang et al. [16] The representative photographs were shown in Figures 4(a) and 4(b). Among the glioma specimens, $102(79.7 \%)$ glioma specimens were positively stained for MPG, whereas its immunoreactivities in nonneoplastic brain sections ranged from undetectable to low (Figure 4(c)). Additionally, the immunostaining of MPG in nonneoplastic brain tissues is below detection levels of both real-time PCR and Western blot. According to the statistical analysis, the expression level of MPG protein in glioma tissues was significantly higher $(P<0.001)$ than that in nonneoplastic brain tissues, which was consistent with the results of Western blot analysis.

In addition, MPG expression was not significantly affected by the gender and age (both $P>0.05$ ) of the patients. In contrast, the MPG expression was the closely correlated with WHO grade, as well as KPS. The expression levels of MPG protein in glioma tissues with higher WHO grade (Table $1 ; P=0.002$ ) and lower KPS (Table $1 ; P=0.01$ ) were significantly higher than those with lower WHO grade and higher KPS.

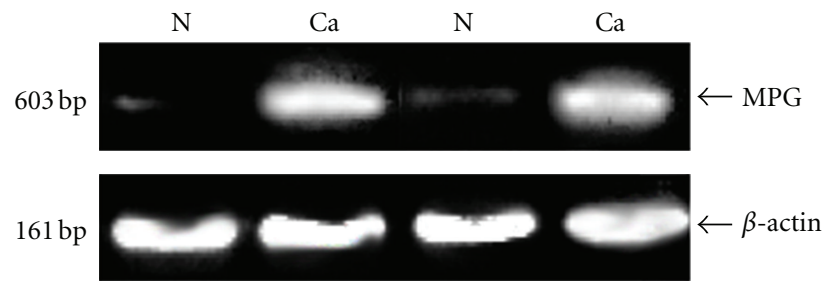

(a)

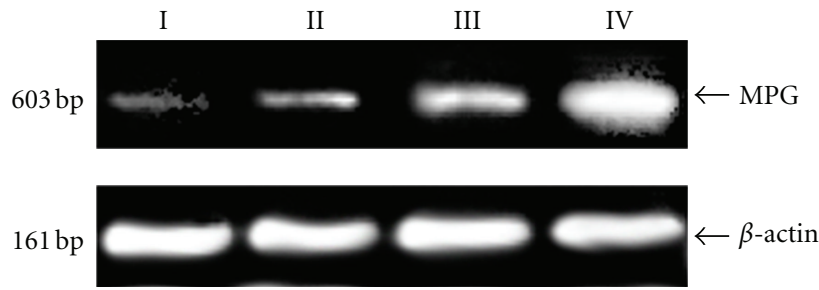

(b)

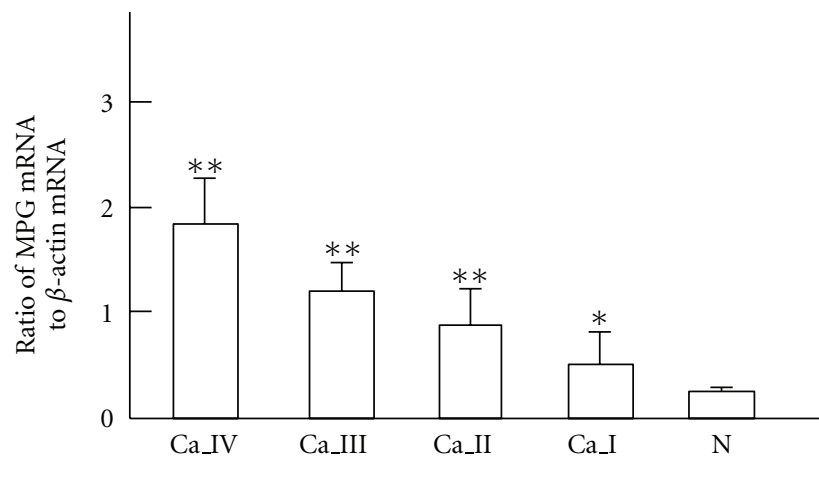

(c)

Figure 2: Expression of MPG gene in glioma and nonneoplastic brain tissues by quantitative real-time PCR analysis. (a) MPG gene expression levels in glioma (lanes 2 and 4) and nonneoplastic brain tissues (lanes 1 and 3). (b) MPG gene expression levels in glioma with different WHO grades. Lanes 1 to 4: MPG gene expression in glioma tissues with WHO grade I, II, III, and IV, respectively. (c) MPG gene expression levels in nonneoplastic brain tissues and glioma with different WHO grades. "N" refers to nonneoplastic brain tissues; "Ca" refers to glioma tissues; "Ca_I" "Ca_IV" refer to glioma tissues with WHO grade I IV. $\beta$-actin was used as an internal control for quantitative real-time PCR analysis. Values are means \pm SD. ${ }^{*} P<0.05$, comparison with nonneoplastic brain tissues; ${ }^{*} P<0.001$, comparison with nonneoplastic brain tissues.

Moreover, we reviewed clinical information of these MPG-positive or -negative glioma patients. During the follow-up period, 100 of the 128 glioma patients $(78.1 \%)$ had died (24 from the MPG-negative group and 76 from the MPG-positive group). As determined by the log-rank test, the survival rate of patients with positive MPG staining was lower than those without MPG staining $(P<0.001$; Figure 5(a)). The median survival time of patients with negative expression of MPG could not be estimated by statistical analysis because all patients survived better than the overall median level, and those patients with strong positive $(+++)$, moderate positive $(++)$, and weak positive 


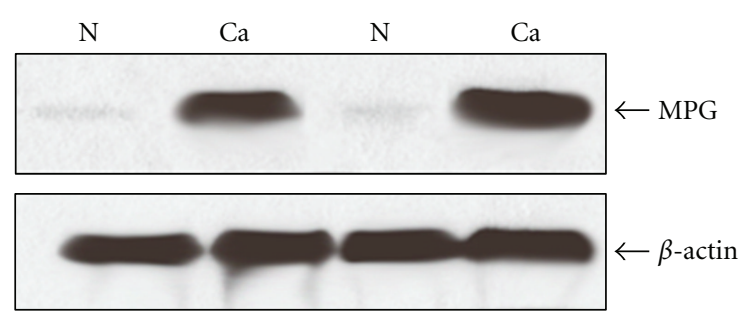

(a)

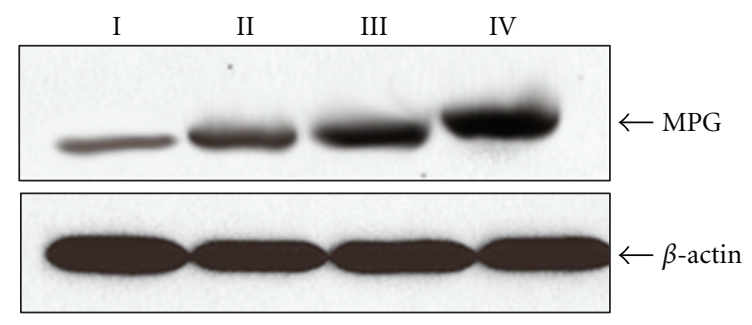

(b)

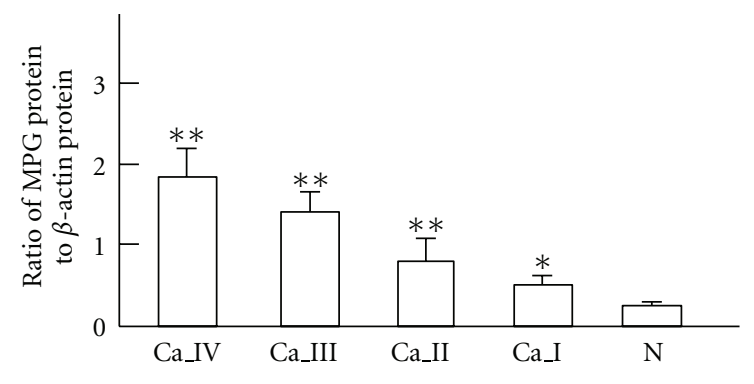

(c)

FIgURE 3: Expression of MPG protein in glioma and nonneoplastic brain tissues by Western blot analysis. (a) MPG protein expression levels in glioma and nonneoplastic brain tissues. (b) MPG expression levels in glioma with different WHO grades. (c) MPG expression levels in nonneoplastic brain tissues and glioma with different WHO grades. "N" refers to nonneoplastic brain tissues; "Ca" refers to glioma tissues; "Ca_I" "Ca_IV" refer to glioma tissues with WHO grade I IV. $\beta$-actin was used as a control for equal protein loading. Values are means \pm SD. ${ }^{*} P<0.05$, comparison with nonneoplastic brain tissues; ${ }^{*} P<0.001$, comparison with nonneoplastic brain tissues.

$(+)$ of MPG were $9.1 \pm 1.5$ months, $12.3 \pm 1.1$ months, and $22.6 \pm 2.2$ months (log-rank test: $P<0.001)$.

Furthermore, Figure 5(b) shows the postoperative survival curve of patients with glioma and MPG expression after adjusting for age, gender, WHO grade, and KPS. By multivariate analysis (Table 2), the loss of MPG expression was a significant $(P<0.001)$ and independent prognostic indicator for patients with glioma besides age, WHO grade and KPS. The Cox-proportional hazards model showed that MPG over-expression was associated with poor overall survival.

\section{Discussion}

Because of the dismal survival in the patients with glioma, especially in GBM patients, there is an urgent need to find
TABLE 2: Cox-multivariate analysis.

\begin{tabular}{lccc}
\hline Parameter & Risk ratio & $\begin{array}{c}95 \% \text { confidence } \\
\text { interval }\end{array}$ & $P$ \\
\hline Age & 0.89 & $0.58-1.65$ & 0.71 \\
Gender & 1.02 & $0.66-1.83$ & 0.33 \\
KPS & 1.99 & $1.28-2.95$ & 0.06 \\
WHO grade & 3.63 & $2.28-7.59$ & 0.03 \\
Extent of resection & 1.29 & $0.89-2.13$ & 0.11 \\
MPG expression & 6.75 & $2.06-15.38$ & $<0.001$ \\
\hline
\end{tabular}

novel specific and effective prognostic markers in order to apply individualized treatments to specifically target the pathophysiologic and molecular properties of glioma patients. In the current study, we investigated the expression of MPG in 128 cases of human glioma and compared the expression with tumor grade and survival rates of patients. Our data demonstrated that MPG gene and protein were both increased in glioma compared to nonneoplastic brain tissue. We found an increased trend of both MPG protein level and gene level from WHO grade I to WHO grade IV glioma. These results suggest that the transcriptional and translational activation of human MPG might participate in the tumorigenesis and progression of glioma.

DNA in all somatic and germ cells in the body are continuously exposed to exogenous and endogenous alkylating and oxidative agents that result in damage to DNA. If DNA damage was not repaired, it may lead to genetic mutation, chromosome aberration, aging, and carcinogenesis. Thus, DNA repair is essential for survival of life. The lack of precise DNA repair activities may lead to defective embryogenesis, tissue-specific dysfunction, hypersensitivity to DNA-damaging agents, premature senescence, genetic instability, and elevated cancer rates. The BER pathway is one of the predominant pathways for DNA repair. The repair of DNA bases damaged by alkylation is initiated in mammalian cells by MPG, as an alkyladenine DNA glycosylase [21]. The majority of repair that is initiated by MPG occurs via short-patch BER, a mechanism whereby only one nucleotide is replaced. MPG has broad specificity for a number of damaged DNA bases. The majority of base damage induced by methylation is removed by MPG, including the principal adducts 3-meA and 7-meG [22]. It has been reported that the expression of MPG could be induced by viral damage and a variety of DNA-damaging agents. For example, the increased expression of MPG gene was studied in breast cancer, cervical neoplasia, and thymic carcinoma in SV $40 \mathrm{~T}$-antigen-expressing transgenic mice [23]. As previous investigation, mammalian cells actively regulated DNA repair enzymes and genes during cell proliferation. DNA repair enzymes are expressed in the cell cycle in a defined temporal pattern relative to the induction of DNA replication. In brain cells that are not undergoing DNA replication, DNA repair is maintaining nucleotide sequences of genomic DNA over time. Kim et al. [24] indicated that MPG expression in the brain was relative high in 1 week after birth, and the level 

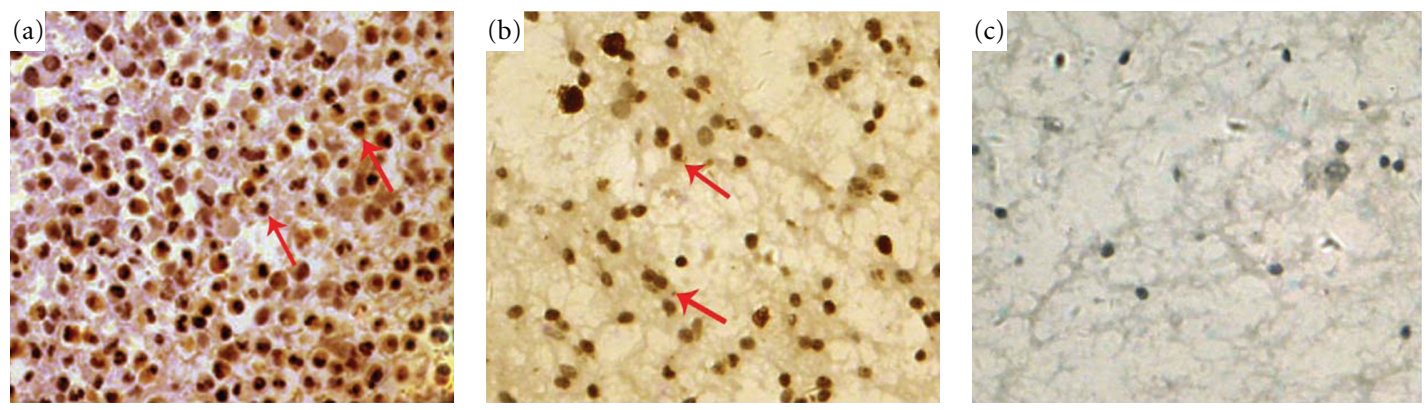

FIGURE 4: Immunohistochemical staining of MPG protein in tumor cells of GBM (a) and astrocytoma (b) (original magnification $\times 200$ ). Staining for this antigen is described in Materials and Methods. Positive staining of MPG is seen in the nuclei of tumors cells and is more abundant in the high- (a) than the low-grade (b) tumors. Negative expression of MPG (c) was observed in nonneoplastic brain tissues.

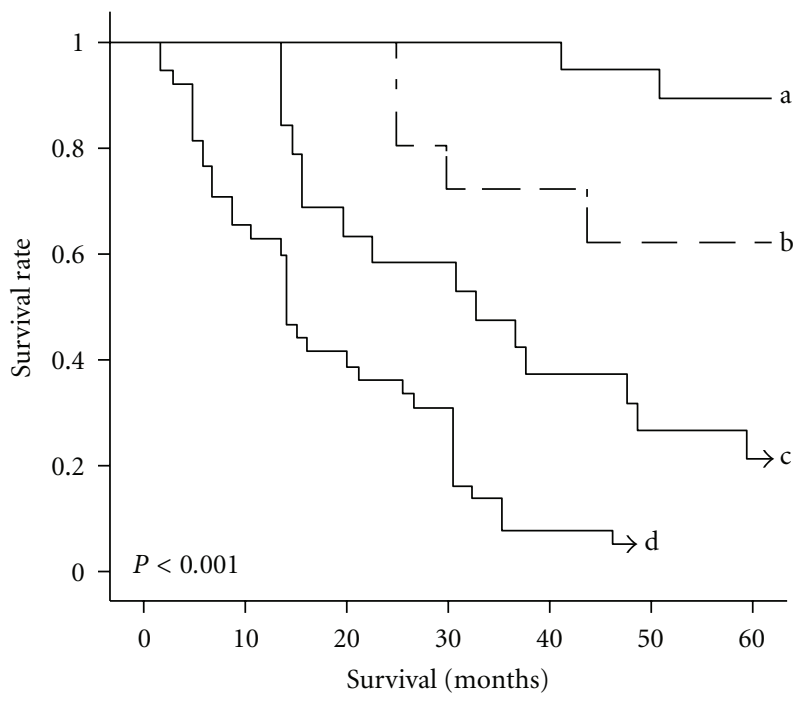

(a)

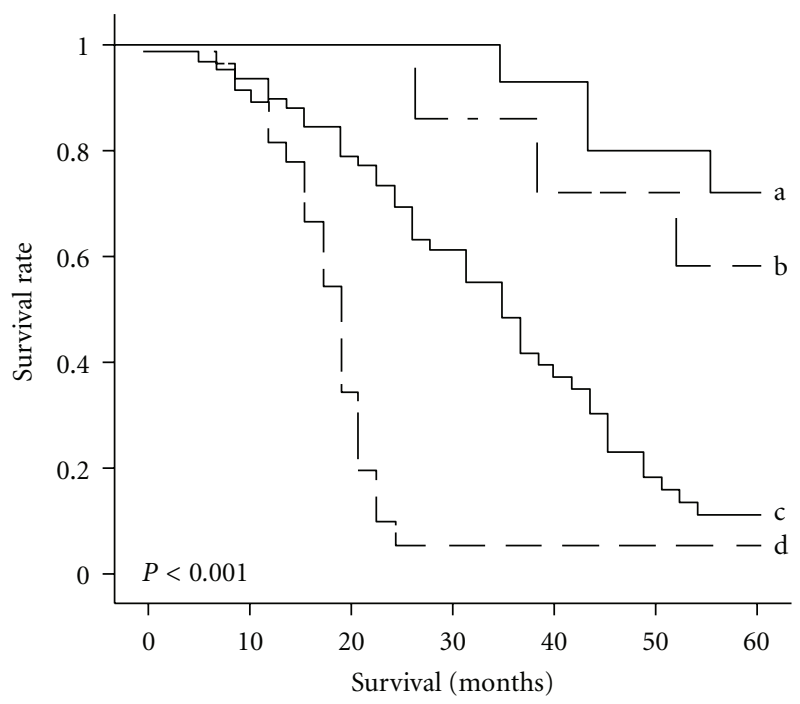

(b)

FIGURE 5: Postoperative survival curves for patterns of patients with glioma and MPG expression. (a) Kaplan-Meier postoperative survival curve for patterns of patients with glioma and MPG expression. (b) Cox-proportional hazards model after adjusting for age, gender, and grade. MPG might be an independent predictor of survival, without consideration of age, gender or grade. "a" refers to MPG-negative expression group; "b" refers to MPG-weak positive $(+)$ expression group; "c" refers to MPG-moderate positive $(++)$ expression group; "d" refers to MPG strong positive $(+++)$ expression group.

remained low in day 400 mature adults, suggesting that brain tissue is terminally differentiated and nonproliferating tissues.

It has been demonstrated that DNA repair enzymes play an important role in the carcinogenesis of several cancers. Of our interests, MPG was overexpressed in breast cancer up to 24-fold as compared to normal primary breast epithelium, suggesting the role of MPG in breast carcinogenesis [12]. In astrocytomas, Kim et al. [18] in 2003 reported that MPG mRNA level was significantly higher than that in tumoradjacent brain tissues. With the similar results, we in this study also found the upregulation of MPG protein and gene in glioma tissues. Additionally, we provide evidence that MPG protein is found in nuclear localization of the glioma tissues, which was consistent with the results of Kim et al. [18]. The nuclear localization of MPG in tumor cells of glioma tissues may be suggestive of a role of MPG in the regulation of cell division and cell cycle. Furthermore, the most important finding of this study was the correlation of MPG expression and survival rates of patients. Our data indicated that nearly $80 \%$ of glioma cases showed positive staining for MPG. The survival rate of patients with positive MPG staining was lower than those without. Kaplan-Meier and multivariate analysis both showed a significantly worse overall survival for patients whose tumors had high MPG levels, indicating that high MPG protein level is a marker of poor prognosis for patients with gliomas. This is the first investigation to demonstrate the prognostic value of MPG in human cancers. Further validations with larger sample size and different methodologies are warranted.

In conclusion, our data showed the over-expression of MPG gene and protein in human gliomas and also suggested for the first time that MPG be an unfavorable independent prognostic indicator for glioma patients. 


\section{Conflict of Interests}

The authors declare that they have no conflict of interests.

\section{Authors' Contribution}

The first three authors offer equal contribution to this paper.

\section{Acknowledgment}

This work was funded by Natural Science Foundation of China (no. 81101736).

\section{References}

[1] H. Ohgaki and P. Kleihues, "Epidemiology and etiology of gliomas," Acta Neuropathologica, vol. 109, no. 1, pp. 93-108, 2005.

[2] D. N. Louis, H. Ohgaki, O. D. Wiestler et al., “The 2007 WHO classification of tumours of the central nervous system," Acta Neuropathologica, vol. 114, pp. 97-109, 2007.

[3] Y. G. Mu, M. Z. Chen, Z. P. Chen, W. N. Zhou, X. H. Zhang, and K. Sai, "Microsurgical technique of brain glioma-a report of 183 cases," Ai Zheng, vol. 23, no. 11, pp. 1317-1321, 2004.

[4] C. Y. Chang, M. C. Li, S. L. Liao, Y. L. Huang, C. C. Shen, and H. C. Pan, "Prognostic and clinical implication of IL-6 expression in glioblastoma multiforme.," Journal of Clinical Neuroscience, vol. 12, no. 8, pp. 930-933, 2005.

[5] E. R. Laws, I. F. Parney, W. Huang et al., "Survival following surgery and prognostic factors for recently diagnosed malignant glioma: data from the glioma outcomes project," Journal of Neurosurgery, vol. 99, no. 3, pp. 467-473, 2003.

[6] H. Lin, Y. Wang, X. Zhang, B. Liu, W. Zhang, and J. Cheng, "Prognostic significance of kappaB-Ras1 expression in gliomas," Medical Oncology. In press.

[7] S. Adhikari, S. Choudhury, P. S. Mitra, J. J. Dubash, S. P. Sajankila, and R. Roy, "Targeting base excision repair for chemosensitization," Anti-Cancer Agents in Medicinal Chemistry, vol. 8, no. 4, pp. 351-357, 2008.

[8] R. N. Trivedi, X. H. Wang, E. Jelezcova, E. M. Goellner, J. B. Tang, and R. W. Sobol, "Human methyl purine DNA glycosylase and DNA polymerase $\beta$ expression collectively predict sensitivity to temozolomide," Molecular Pharmacology, vol. 74, no. 2, pp. 505-516, 2008.

[9] J. F. Harrison, M. L. Rinne, M. R. Kelley, N. M. Druzhyna, G. L. Wilson, and S. P. Ledoux, "Altering DNA base excision repair: use of nuclear and mitochondrial- targeted N-methylpurine DNA glycosylase to sensitize astroglia to chemotherapeutic agents," Glia, vol. 55, no. 14, pp. 1416-1425, 2007.

[10] B. Kaina, G. Fritz, and T. Coquerelle, "Contribution of O6alkylguanine and $\mathrm{N}$-alkylpurines to the formation of sister chromatid exchanges, chromosomal aberrations, and gene mutations: new insights gained from studies of genetically engineered mammalian cell lines," Environmental and Molecular Mutagenesis, vol. 22, no. 4, pp. 283-292, 1993.

[11] S. K. Randall, R. Eritja, B. E. Kaplan, J. Petruska, and M. F. Goodman, "Nucleotide insertion kinetics opposite abasic lesions in DNA," Journal of Biological Chemistry, vol. 262, no. 14, pp. 6864-6870, 1987.

[12] M. L. Fishel, Y. R. Seo, M. L. Smith, and M. R. Kelley, "Imbalancing the DNA base excision repair pathway in the mitochondria; targeting and overexpressing N-methylpurine DNA glycosylase in mitochondria leads to enhanced cell killing," Cancer Research, vol. 63, no. 3, pp. 608-615, 2003.

[13] S. R. Cerda, P. W. Turk, A. D. Thor, and S. A. Weitzman, "Altered expression of the DNA repair- protein, Nmethylpurine-DNA glycosylase (MPG), in breast cancer," The FEBS Letters, vol. 431, no. 1, pp. 12-18, 1998.

[14] T. J. Sohn, N. K. Kim, H. J. An et al., "Gene amplification and expression of the DNA repair enzyme, N-methylpurineDNA glycosylase (MPG) in HPV-infected cervical neoplasias," Anticancer Research, vol. 21, no. 4, pp. 2405-2411, 2001.

[15] M. L. Fishel, Y. He, M. L. Smith, and M. R. Kelley, "Manipulation of base excision repair to sensitize ovarian cancer cells to alkylating agent temozolomide," Clinical Cancer Research, vol. 13, no. 1, pp. 260-267, 2007.

[16] D. Wang, Z. Y. Zhong, Q. H. Zhang, Z. P. Li, and M. R. Kelley, "Effect of adenoviral N-methylpurine DNA glycosylase overexpression on chemosensitivity of human osteosarcoma cells," Chinese Journal of Pathology, vol. 35, no. 6, pp. 352-356, 2006.

[17] M. Rinne, D. Caldwell, and M. R. Kelly, "Transient adenoviral N-methylpurine DNA glycosylase overexpression imparts chemotherapeutic sensitivity to human breast cancer cells," Molecular Cancer Therapeutics, vol. 3, no. 8, pp. 955-967, 2004.

[18] N. K. Kim, J. Y. Ahn, J. Song et al., "Expression of the DNA repair enzyme, N-methylpurine-DNA glycosylase (MPG) in astrocytic tumors," Anticancer Research, vol. 23, no. 2, pp. 1417-1423, 2003.

[19] J.-B. Tang, D. Svilar, R. N. Trivedi et al., "N-methylpurine DNA glycosylase and DNA polymerase $\beta$ modulate BER inhibitor potentiation of glioma cells to temozolomide," Neuro-Oncology, vol. 13, no. 5, pp. 471-486, 2011.

[20] M. M. Bradford, "Rapid and sensitive method for the quantitation of microgram quantities of protein utilizing the principle of protein-dye binding," Analytical Biochemistry, vol. 72, no. 1-2, pp. 248-254, 1976.

[21] N. K. Kim, H. J. An, H. J. Kim et al., "Altered expression of the DNA repair protein, N-methylpurine-DNA glycosylase (MPG) in human gonads," Anticancer Research, vol. 22, no. 2, pp. 793-798, 2002.

[22] M. L. Rinne, Y. He, B. F. Pachkowski, J. Nakamura, and M. R. Kelley, "N-methylpurine DNA glycosylase overexpression increases alkylation sensitivity by rapidly removing non-toxic 7-methylguanine adducts," Nucleic Acids Research, vol. 33, no. 9, pp. 2859-2867, 2005.

[23] N. K. Kim, S. H. Lee, K. Y. Cha, and J. S. Seo, "Tissue-specific expression and activation of N-methylpurine-DNA glycosylase in thymic carcinomas of transgenic mice expressing the SV40 large T-antigen gene," Molecules and Cells, vol. 8, no. 4, pp. 383-387, 1998.

[24] N. K. Kim, S. H. Lee, T. J. Sohn et al., "Spatial expression of a DNA repair gene, $\mathrm{N}$-methylpurine-DNA glycosylase (MPG) during development in mice," Anticancer Research, vol. 20, no. 5, pp. 3037-3043, 2000. 

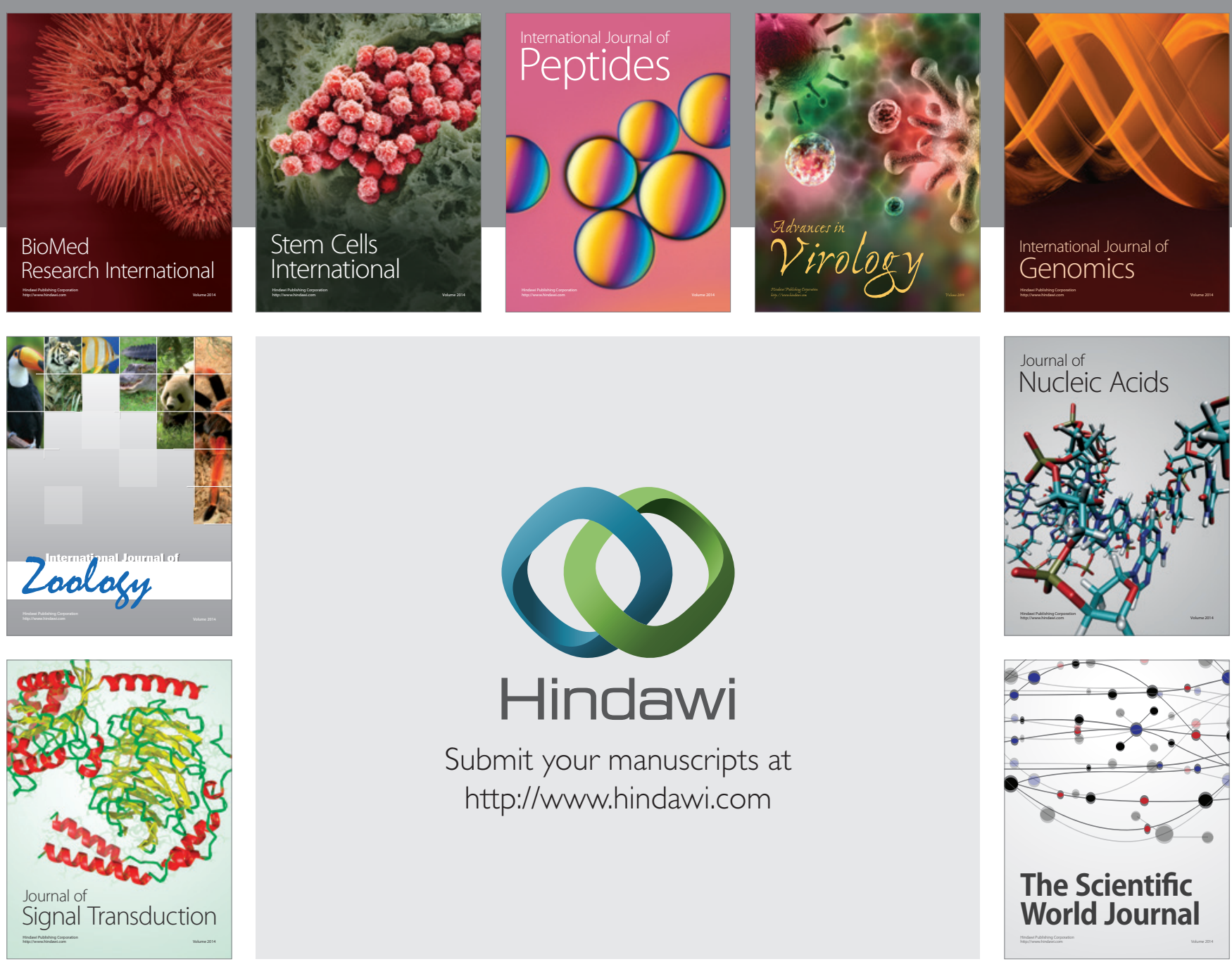

Submit your manuscripts at

http://www.hindawi.com
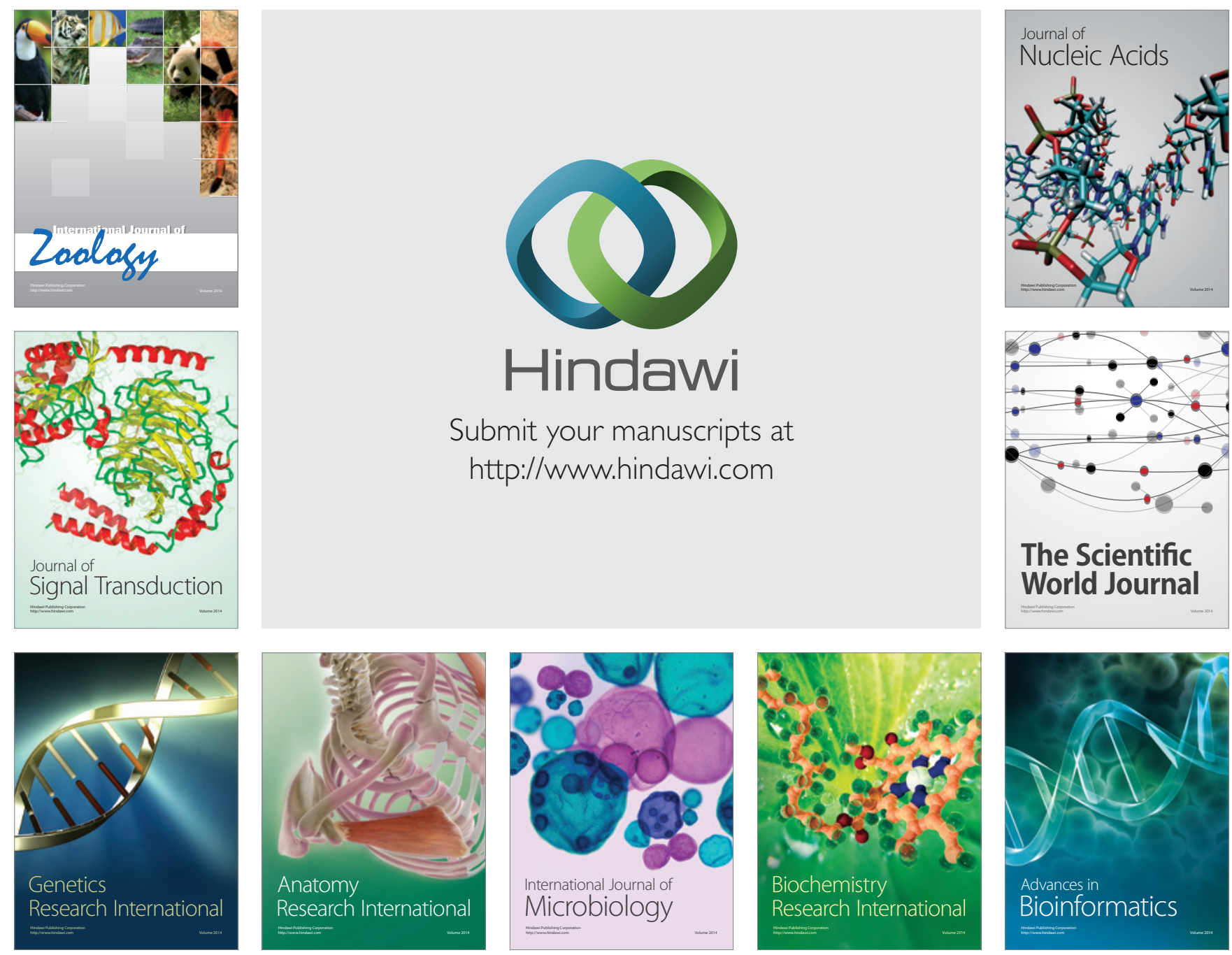

The Scientific World Journal
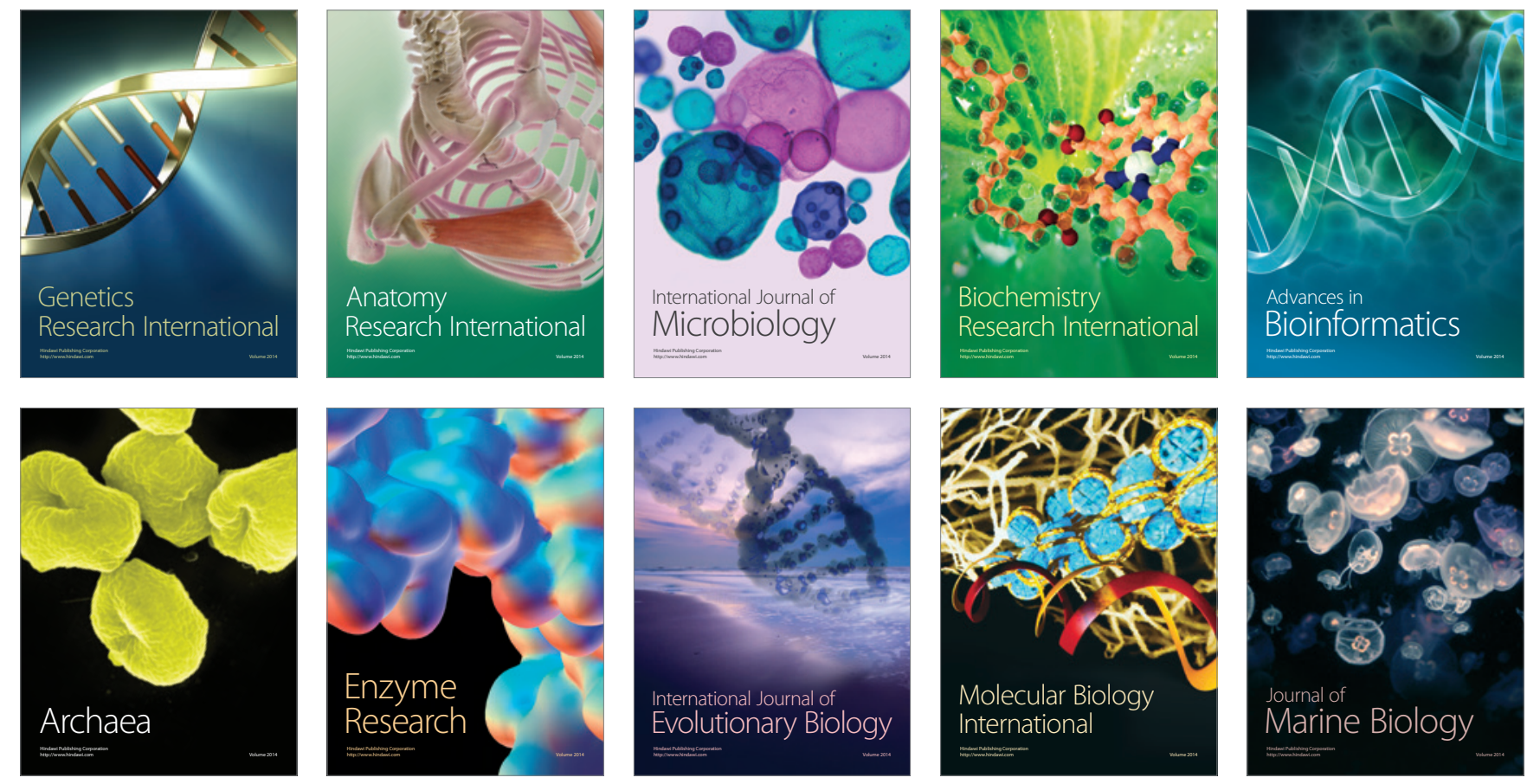